\title{
MUJER EN LA GOBERNANZA UNIVERSITARIA Y SUS PRINCIPALES BARRERAS
}

\section{WOMEN IN UNIVERSITY GOVERNANCE AND ITS MAIN BARRIERS}

\author{
Ivette Marité DURÁN-SEGUEL ${ }^{1}$ \\ Maximiliano GALLEGOS RIVERA ${ }^{2}$ \\ Pricila NAVARRETE CASTRO ${ }^{3}$
}

\begin{tabular}{|lll|}
\hline Recibido & $:$ & 03.12 .2021 \\
Aprobado & $:$ & 27.12 .2021 \\
Publicado & $:$ & 31.12 .2021 \\
\hline
\end{tabular}

\begin{abstract}
RESUMEN: El propósito de esta investigación es analizar los factores que influyen en el bajo nivel de participación de las mujeres en cargos directivos en las universidades chilenas. Para el logro de este objetivo se utiliza un enfoque cualitativo desde el análisis fenomenológico. La información se recolecta a partir de entrevistas semi estructuradas dirigidas a mujeres que forman parte de la dirección de las universidades. Los resultados señalan que existen diversos factores que influyen en la baja participación de las mujeres como las barreras invisibles y las etiquetas culturales que les impiden acceder a estos cargos. El estudio muestra que es necesario la creación de espacios para potenciar la participación de la mujer en cargos de poder en las universidades, que permitan fortalecer el liderazgo femenino compatibilizando las responsabilidades laborales y familiares, a través del rediseño de la carrera académica.
\end{abstract}

Palabras claves: universidades, mujeres alta dirección, educación superior, gobernanza universitaria, barreras de género.

\begin{abstract}
The purpose of this research is to analyze the factors that influence (influencing) the low level of participation of women in managerial positions in Chilean universities. To achieve this goal, a qualitative approach was used from the phenomenological analysis. The information was gathered from semi-structured interviews focused to women who are part of the university leadership. The results point to the existence of a variety of factors that influence the low participation of women such as invisible barriers and cultural labels that prevent them from accessing these positions. The study shows that it is necessary to create spaces to promote the participation of women in positions of power in universities, which allow strengthening female leadership by making work and family responsibilities compatible, through the redesigning of the academic career.
\end{abstract}

Keywords: Universities, women in high position, higher education, university governance, gender barriers.

\footnotetext{
${ }^{1}$ Ingeniero Comercial, Master Business Administration, Doctora en Ciencias de la Administración, Universidad Católica del Maule - Facultad de Ciencias Sociales y Económicas, Departamento de Economía y Administración. Talca, Chile. Email: iduran@ucm.cl ORCID: https://orcid.org/0000-0002-0903-5811

${ }^{2}$ Ingeniero Comercial, Magíster en Dirección Financiera, Universidad de Talca - Facultad de Economía y Negocios. Talca, Chile. Email: mgallegos@utalca.cl (autor de correspondencia). ORCID: https://orcid.org/0000-0001-5926-7488

${ }^{3}$ Ingeniero Comercial, Licenciada en Administración, Universidad Católica del Maule - Facultad de Ciencias Sociales y Económicas, Talca, Chile Email: pricilanavarrete@hotmail.es ORCID: https://orcid.org/0000-0002-7833-951X
} 


\section{INTRODUCCIÓN}

Las desigualdades que imperan en nuestra sociedad, han sido el sostén de las relaciones de género, derivadas de constructos sociales, que focalizan la discriminación en la mujer, quien ha sido vulnerada en sus derechos por su sola condición de mujer (Méndez y Rojas, 2014). En términos laborales, a través del tiempo a la mujer se le ha asignado un rol reproductivo y doméstico, en tanto que al hombre se le posiciona en el ámbito productivo y despeñándose fuera del hogar. No obstante, en la última década se han dado importantes cambios (Adu-Oppong et al., 2017), debido a que el papel de la mujer en el mercado laboral se ha visto incrementado por su mayor capacitación y desarrollo profesional (Matteo, 2012). De esta manera, podemos notar una creciente participación de las mujeres en diferentes áreas, especialmente en las universidades en donde forman la mayor parte del colectivo del alumnado (Duarte, 2012).

La pandemia ha desmejorado la situación de las mujeres en el ámbito laboral, a nivel mundial, sin embargo, el desequilibrio tanto en la fuerza laboral como en los cargos directivos ocupados por mujeres, es un tema que ha sido preocupación y objeto de estudio desde hace varios años. Si bien, no se puede desconocer el sostenido aumento de la escolaridad y el creciente ingreso de las mujeres a las universidades (Papadópulos y Radakovich, 2006; Ricks, 2018), aun así, queda bastante camino por recorrer para alcanzar la igualdad y equidad de género, particularmente en el liderazgo de las mujeres en diferentes ámbitos, tales como el mundo privado, público y en educación superior (Peiying y Hsiao-chin, 2019).

\section{CONTEXTUALIZACIÓN}

\section{Género}

El género tiene diferentes contribuciones teóricas y examina como las sociedades, en la relación de hombres y mujeres, construyen valores, reglas, costumbres, prácticas y subjetividades (Del Pino, 2015). Esta relación, asume una diferencia de roles en sociedad, los cuales son un constructo cultural, y no una condición biológica o natural (Gaete y Álvarez, 2020). En la literatura de género, se expone que la presencia de mujeres en cargos de responsabilidad y poder aún es baja, predominando los hombres en puestos de mayor responsabilidad, lo anterior ha derivado en lo que denominamos "techo de cristal", una pared invisible pero que frena el acceso a un piso superior, 


\section{Journal of the Academy | 32 |}

por medio de procedimientos, con relaciones de poder y creencias, que sin duda, obstaculiza el ingreso de mujeres a puestos de poder, toma de decisiones y liderazgo (Gutiérrez, 2015; AduOppong, et al., 2017; Gaete y Álvarez, 2020). Lo anterior implica que las mujeres se ven obligadas y quedan relegadas a puestos de trabajo menos valorados, reconocidos y con salarios más bajos.

El techo es invisible, lo que implica que no es percibido como un freno para el desarrollo de las mujeres, por gran parte de la sociedad y porque además, no existen elementos de discriminación explicita en contra de las mujeres, porque principalmente deriva de determinados comportamientos sociales y culturales (Matus y Gallego, 2015). El techo de cristal, se ha utilizado como un marco de reflexión en relación a las causas que generan una sub-representación de la mujer en altos niveles organizacionales (Gaete, 2015). Lo anterior, derivado de prejuicios que funcionan como una barrera que no les permite a las mujeres seguir escalando a posiciones de más alto nivel, quedando atrapadas en niveles medios de la dirección (Camarena y Saavedra, 2018). Pero, no solo el techo de cristal influye en el proceso de marginación continuada hacia las mujeres que acontece en los entornos laborales, sino que también está la existencia de barreras internas que se asocian a la identidad del género femenino es decir, a características y actitudes asociadas a las mujeres las cuales pueden ser negativas para el éxito profesional; y las barreras externas que se asocian a la cultura organizacional, que puede incidir negativamente, en la promoción laboral de las mujeres como consecuencia de procesos viciados por estereotipos y roles de género (Barberá et al., 2011).

Las mujeres no solo se enfrentan al techo de cristal, sino también a la metáfora asociada al suelo pegajoso, este constructo ilustra los problemas a los que se ven enfrentadas las mujeres profesionales, al momento de acceder a los primeros niveles de la carrera académica (Torres y Pau, 2011). Esta metáfora ejemplifica que las mujeres caminan sobre un suelo, que no les permite despegarse del mismo, y tener la libertad de llegar a ocupar mejores posiciones directivas y de poder en las organizaciones (Del Pino, 2015). Además, se refiere a la existencia de obstáculos en relación con las tareas familiares y proveedores de atención (Gallego, 2017). Así se establece que se produce una exclusión de las mujeres a los altos cargos de cualquier tipo de organización. Según McPherson (2010), las barreras para las mujeres en la alta dirección, se pueden dividir en tres categorías que interactúan: individuales, organizacionales y factores culturales, sociales o externos más amplios. Las razones individuales que se transforman en una barrera se asocian a una menor proactividad en la búsqueda de posiciones de poder, perfeccionismo excesivo, las mujeres tienden 


\section{Journal of the Academy | 33 |}

a mirarse con estereotipos culturales, lo que muchas veces afecta su autoestima. Por otra parte, entre las razones organizacionales se cuentan, una cultura corporativa dominantemente masculina y redes informales, prejuicios hacia las mujeres, falta de oportunidades de desarrollo; lugares de trabajo inflexibles en el diseño de puestos y trayectorias profesionales; acoso y la discriminación. Finalmente, los factores culturales, donde se incluyen el marco legislativo, el contexto informal de las normas sociales en relación con la equidad de género, así como el ámbito doméstico de la familia y el hogar (McPherson, 2010).

\section{Liderazgo y género}

El concepto de líder se ha desarrollado en diversos artículos siendo uno de los temas más estudiados las últimas décadas (Dinh et al., 2014). Así como lo definen Vásquez et al., (2014), los líderes tienen la capacidad de movilizar e influir a las personas hacia una dirección e integrarlos en un proyecto común. En el último tiempo, se ha profundizado en el estudio de dos enfoques de liderazgo (Serrano y Portalanza, 2014; García y Lopez, 2006), el liderazgo transformacional y el liderazgo transaccional. El primero se dirige a reforzar valores y creencias, generando una imagen de confianza que permite que se sientan identificados y tiendan a imitarlos (Maak et al., 2016; Lupano y Castro, 2013; Diaz et al., 2019; Durán-Seguel, et al., 2019) y, por otro lado, el liderazgo transaccional se caracteriza por retribuir de manera positiva, con un intercambio generalmente de índole económico o de manera negativa a través de castigos (Mendoza et al., 2012). Sin embargo, es importante mencionar que se sostiene que ambos liderazgos no son excluyentes y que se permite ambos liderazgos en las organizaciones dependiendo de las situaciones que se presenten (Lupano y Castro 2013). En este ámbito, podemos hacer la relación entre estilos de liderazgos y género, donde diversas investigaciones han llegado a la conclusión de que las mujeres desempeñan un liderazgo más cercano a lo transformacional, siendo por el contrario el liderazgo transaccional asociado a los hombres (Vinkenburg et al., 2011; Hernandez et al., 2014; Brandt y Edinger, 2015; Moncayo y Pinzón, 2013). Complementando la información anterior, también existen una serie de autoras, que argumentan que las mujeres tienen estilos de liderazgo asociado a su género, denominándolo "femenino" (García y Lopez, 2006; Perozo y Paz, 2016). Existen características particulares asociadas a este estilo, como es la cooperación, colaboración entre el líder y sus colaboradores, un menor control del líder y resolución de problemas que se basan en la intuición, empatía y racionalidad (Perozo y Paz, 2016). Al considerar que hombres y mujeres tienen 


\section{Journal of the Academy | 34 |}

diferentes formas de liderar una organización (Gallegos et al., 2012), y que generalmente las características que se asocian al rol de líder se orientan frecuentemente al rol de género masculino más que al femenino (García y Lopez, 2006), es natural que se pueden apreciar grandes brechas de género en el mercado laboral, que atentan contra las condiciones de igualdad entre ambos sexos (Vaca, 2019), esto tiene directa relación con una menor representación de mujeres en altos puestos directivos. Además, bajo esta premisa, cuesta más que una mujer pueda ser aceptada como líder por sus subordinados (Dezsö y Gaddis, 2007).

\section{El liderazgo de las mujeres en el contexto de Educación Superior}

Las universidades son organizaciones donde se cultiva el saber, cuna de las grandes transformaciones de la sociedad, con cambios de mentalidad y aportando avances al mundo. En consecuencia, podría esperarse que en una organización que promueve la valoración intelectual por, sobre todo, el avance en ámbitos profesionales experimentados por las mujeres esté en concordancia con una mayor participación en la gestión directiva universitaria (Del Pino, 2015; Adu-Oppong, et al., 2017). Sin embargo, a pesar del mayor nivel educativo que ostentan quienes trabajan en las universidades, las mujeres enfrentan obstáculos para asumir roles de alta dirección. Este fenómeno se da a nivel global, donde sólo hay un 36\% de mujeres en roles de liderazgo (Foro Económico Mundial, 2020), las mujeres están subrepresentadas en la alta dirección, y su aceptación como líderes es menor en relación a los hombres, problema que se acrecienta en los países en desarrollo (Maheshwari y Nayak, 2020). Las mujeres que son líderes se deben enfrentar a diferentes barreras que le impiden avanzar en su carrera, las cuales pueden incluir factores organizacionales, estructurales, culturales o personales (Maheshwari y Nayak, 2020).

En la educación superior, y específicamente en universidades, se esperaría una mayor participación femenina en cargos directivos, ya que son este tipo de organizaciones donde debiese primar el intelecto por sobre el género, sin embargo, persiste un desequilibrio de género y subrepresentación de las mujeres en cargos directivos (Wylie, et. al, 2007; Sheperd, 2017; Maheshwari y Nayak, 2020). Particularmente, en el caso de Chile, solo un 4\% de los cargos de rectorías de universidades estatales y católicas (objeto de estudio de esta investigación) están ocupados por mujeres. Según el Foro de educación superior (Aequalis, 2017) solo el 20,5\% de los cargos de rectorías o vicerrectorías estaban en manos femeninas, en la actualidad estas cifras han aumentado a un 29\%, siendo aún muy descendidas. En relación a los altos cargos en los Consejos Superiores o juntas 


\section{Journal of the Academy | 35 |}

directivas, dentro de las universidades chilenas, las mujeres sólo alcanzan una participación de un $31 \%$ (información obtenida de los sitios web de las universidades), y es allí donde la igualdad y el acceso a cualquier posición de poder dentro de las universidades comienza a cuestionarse. Por ello, es importante analizar particularmente este nicho, ya que las juntas directivas son las que toman las decisiones estratégicas en las universidades.

Las investigaciones sobre cómo abordar las cuestiones de género han aumentado recientemente y han investigado las barreras para que las mujeres accedan a puestos de liderazgo en las universidades (Aslanargun, 2013), sin embargo, aún persiste su baja participación. Aunque las mujeres directivas tienen altos niveles de educación y deseo de progresar en sus carreras académicas, sigue siendo cierto que pocas alcanzan el mismo estatus que sus homólogos masculinos, incluso en las economías avanzadas (Adu-Oppong, et al., 2017; Maheshwari y Nayak, 2020).

\section{METODOLOGÍA}

La presente investigación es de tipo cualitativo en base a un análisis Fenomenológico. Esta metodología busca describir el significado de las experiencias vividas por varios individuos sobre un concepto o fenómeno (Creswell, 2007). La recopilación de datos se realizó utilizando una muestra de tipo no probabilístico intencional (Tongco, 2007), el estudio fue enfocado particularmente a mujeres que son parte de las juntas directivas en las universidades católicas pertenecientes al Consejo de Rectores $(\mathrm{CRUCH})$ y universidades estatales chilenas, se excluyeron las universidades privadas y las no pertenecientes al CRUCH debido a la falta de tiempo y la dificultad para acceder a la información de estas instituciones. Se utilizó como instrumento de recopilación de los datos, entrevistas semi estructuradas aplicadas a nueve consejeras. La estructura y preguntas de la entrevista fueron validadas anteriormente, a través de una prueba preliminar. La operacionalización de la entrevista se realizó por medio de datos oficiales de cada casa de estudios, contactándose a las consejeras por medio de correo electrónico y medios electrónicos. Las entrevistas fueron realizadas por zoom y teams, y tuvieron una duración promedio de 45 minutos aproximadamente. 
El objetivo del estudio fue dilucidar la siguiente pregunta de investigación: ¿Cuáles son los factores que influyen en la baja participación de las mujeres en la alta dirección en la educación superior, específicamente las juntas directivas de las universidades chilenas?

\section{RESULTADOS}

Los resultados obtenidos nacen a partir de la aplicación del instrumento cualitativo, analizando las variables asociadas a la investigación, considerando las percepciones, respuestas y opiniones de las entrevistadas. Responden al objetivo general de la investigación de analizar los factores que influyen en la baja participación de las mujeres en cargos de la junta directiva de las universidades chilenas. En primer lugar, se presentarán las principales características que poseen en común las entrevistadas, y luego se analizarán las barreras que están impidiendo el avance de la mujer en la participación de las altas esferas de decisión en las universidades chilenas.

\section{Caracterización de las entrevistadas juntas directivas de las universidades chilenas.}

Una de las características comunes de las entrevistadas es que llevan más de un período de ejercicio en su rol de consejera, la mayoría se encuentra en su segundo y tercer periodo, por lo tanto, se puede apreciar que la disminuida motivación de las mujeres a participar en la junta directiva, implica que además de existir baja representación se deban repetir las mismas personas en los cargos. Otra característica relevante, es que todas estudiaron en colegios municipales femeninos, universidades estatales, ocho de las nueve entrevistadas poseen posgrado y todas se desempeñaron en diferentes cargos directivos mientras estudiaban, en la educación secundaria o universitaria. Finalmente, todas las entrevistadas son solteras (2) o tienen hijos que ya finalizaron sus estudios escolares primarios (7).

\section{Barreras para el liderazgo de las mujeres en el contexto de Educación Superior}

\section{Nivel individual}

Este nivel se relaciona con la posición de las propias mujeres, como, por ejemplo, las decisiones centradas en la familia versus trabajo y la maternidad, características relacionadas con la autoestima, auto confianza, empoderamiento, entre otros (McPherson, 2010). 


\section{Journal of the Academy | 37 |}

En las entrevistas aplicadas se pudo ver que principalmente su participación como consejera tiene que ver con un crecimiento personal, con aportar, tener nuevos aprendizajes y además el sentirse honradas y orgullosas por ser electas para participar de dicho Consejo. En todas ellas hay un sentimiento de deber con la representación asignada, un sentido de responsabilidad con quienes las nominaron. También hay una mirada de aportar a través de su participación en las decisiones con una mirada institucional. Además, quieren aportar con nuevas propuestas e ideas.

(comunicación personal E1, 2020) "La junta directiva es el máximo cuerpo colegiado dentro de una universidad y por ende, es sumamente importante tener un cargo dentro de ella", (comunicación personal E3, 2020) "Desde el momento que asumí esta responsabilidad he estudiado, he leído y analizado cada documento que me han enviado, siento que para eso estoy aquí para representar a mis colegas y para dar opiniones y tomar decisiones con fundamento"; (comunicación personal E4, 2020) "La responsabilidad de este cargo es grande estamos tomando decisiones que son de largo plazo e impactarán a generaciones de jóvenes y académicos"; (comunicación personal E7, 2020) "Las ideas y propuestas que nosotras podemos entregar desde la Junta son muy relevantes porque tenemos una mirada diferente que la de un hombre y empatizamos con la mitad del alumnado, y claramente nos complementamos como Consejeros".

En consecuencia, se podría pensar que todas las mujeres quisieran participar en los Consejos o Juntas directivas, lo cual no necesariamente es así, las académicas tienen su opinión respecto de la baja participación de mujeres en las Juntas Directivas. (comunicación personal E8, 2020) "Miedo al riesgo", (comunicación personal E4, 2020) "Por permanecer en la zona de confort", (comunicación personal E6, 2020) "Falta de interés por participar en la toma de decisiones", (comunicación personal E9, 2020) "Falta de habilidades blandas". Estableciendo así que existen tipos de barreras que dificultan el acceso a los altos cargos por parte de las mujeres, sin embargo, destacan también que son ellas mismas las que se colocan estos obstáculos. Por otra parte, también consideran que muchas veces no hay participación porque se quiere evitar un trabajo extra sin compensación económica y por la falta de las capacidades de estar en cargos que implican tomar una decisión (postura) lo cual siempre es necesario para poder desempeñar estos cargos.

(comunicación personal E3, 2020) ... o sea cuantos estudios hay de cuantas mujeres están en cargos gerenciales o sea son las menos... ha costado y a pesar que hemos demostrado que, si lo hacemos mejor, las decisiones son diferentes, ahora también depende de la mujer, porque también hay mujeres que llegan a cargos altos y que no no.... Que pueden tener la capacidad de gestión y todo, pero les falta la capacidad... las habilidades blandas... 
(comunicación personal E5, 2020) "Hay que saber estar en estos cargos... yo siempre digo hay que tener características que no se compran en el almacén de la esquina... son características que tiene que tener una persona... que las trae de mucho antes...”. Luego, es posible apreciar que las entrevistadas favorecen la participación femenina en puestos de liderazgo, siempre y cuando dicha participación se relacione con las capacidades de cada persona.

En relación a la maternidad, este factor se considera desde dos puntos de vista y esto principalmente porque las entrevistadas manifestaron que si se tienen hijos pequeños influye al tomar la decisión si participar o no como Consejera. (comunicación personal E5, 2020) "Claro que influye, por el hecho de que toda mujer prefiere pasar más tiempo con sus hijos pequeños que optar por un cargo que requiere de mucha responsabilidad y tiempo", (comunicación personal E6, 2020) “Cuando uno es mamá implica de algún modo la interrupción de la vida profesional o laboral, contribuyendo al abandono voluntario de sus actividades laborales por un tiempo para dedicarse a la crianza de sus hijos", (comunicación personal E3, 2020) "Optar por cargos que conllevan mayores responsabilidades, en algún momento pasan la cuenta, porque en la maternidad se reflejará como un sentimiento de culpa que muchas veces tienen las mujeres por pasar fuera de casas tantas jornadas laborales, incluyendo viajes". (comunicación personal E1, 2020) "Mira lo ideal es que los niños ya estén crecidos y tengan autonomía, para así responder bien a esta gran tarea que se te encomienda". En general, las consejeras dijeron que no influyó en su decisión, porque o no tenían hijos o éstos eran grandes (cursaban secundaria o universidad) y ellas tenían una trayectoria profesional desarrollada.

\section{Nivel organizacional}

Este nivel está asociado a la carrera académica, tanto desde el punto de vista orgánico, como desde la priorización de los tiempos en términos de la docencia, investigación, extensión y gestión académica. Según la literatura existen decisiones que a veces se toman de manera informal, y en horarios extraordinarios, a los cuales las mujeres no pueden acceder por tener el rol de cuidadora del hogar. Así también existen otras barreras como son comisiones de evaluación académica o de progresión académicas mayoritariamente masculinas, entre otros, (McPherson, 2010). 


\section{Journal of the Academy | 39 |}

Las decisiones dentro de la junta directiva son tomadas a partir de votaciones que son a "mano alzada" o "secretas" dependiendo del tipo de decisión que se requiera tomar, y todos los participantes están en igualdad de condiciones al momento de decidir. Asimismo, las entrevistadas manifiestan que: (comunicación personal E4, 2020) "Yo creo que debería haber más mujeres porque el pensamiento que tiene el hombre es distinto al que tiene la mujer...” En relación a las decisiones, en general las entrevistadas aseveran que éstas no se realizan fuera del horario de trabajo, (comunicación personal E2, 2020) "Si pasa, que muchas veces hay que quedarse fuera del horario de trabajo revisando documentos, pero reuniones, no”.

En relación a la existencia de barreras invisibles la mayoría de las entrevistadas manifestó no haber vivido situaciones laborales que les haya impedido principalmente lograr un buen desarrollo profesional y la no existencia de prejuicios sobre las mujeres en este tipo de cargos, donde ellas manifestaron que: (comunicación personal E8, 2020) "En esta universidad yo creo que no, nunca he sabido de algo así como de prejuicios en contra de nosotras o que tramen algo, no...”

(comunicación personal E2, 2020) Lo que yo he visto en la junta directiva en los cuatro años y algo que llevo en ella, al contrario, yo creo que se valora mucho el aporte de las mujeres, no porque sean mujeres, sino porque las mujeres que llegan ahí suelen ser muy destacadas entonces son un aporte y siempre he visto mucho respeto y mucha valoración...

Por lo tanto, ellas manifiestan recibir un trato similar al de sus pares hombres, en relación al ámbito laboral. No se mencionó que existan atributos que se exijan solo a las mujeres o solo a hombres, sino que a ambos géneros y lo que más destacan es que se debe tener capacidad de coordinación, de liderazgo, una carrera profesional desarrollada y tener la capacidad de trabajar en equipo. En otras palabras, la creencia de que existen puestos de trabajo para mujeres y otros para hombres, está siendo desplazada, teniendo casos, como estos, que muestran que las mujeres pueden estar en distintos cargos de liderazgo, sin tanta dificultad.

En consecuencia, según las entrevistadas, los académicos que pueden pertenecer a la junta directiva son personas con una trayectoria profesional importante y que deben pertenecer principalmente a la alta jerarquía académica. (comunicación personal E7, 2020) "Las actividades que tu desarrollas, como investigación, por docencia, necesitas una trayectoria, una línea de investigación, todas las actividades que uno hace como académica... son evaluadas y ahí uno 


\section{Journal of the Academy | 40 |}

puede ir subiendo de jerarquía, pero demora...”. (comunicación personal E5, 2020) "Es difícil subir en la escala de jerarquización académica, porque cuando uno ha sido mamá demora años en nivelar el tiempo de pre y posnatal, sobre todo porque hay exigencias respecto de investigación, que no es tan fácil de recuperar".

(comunicación personal E3, 2020)" Aquí es donde yo digo que pesa la maternidad, más que en el asumir cargos académicos, sino en tú desarrollo académico, que no necesariamente incide en el cargo, porque yo puedo tener una categoría académica y no tener un cargo o al revés, es en la categorización donde te castiga la institución”.

Si bien, las entrevistadas manifiestan que no sienten que exista discriminación en su elección como consejeras porque siempre se han elegido mujeres, sin embargo, aún son pocas (una o dos en cargos dentro de la junta directiva). Aun así, es en su progresión o desarrollo de carrera académica donde hay mayor desigualdad.

\section{Nivel cultural}

Este nivel se asocia principalmente con la cantidad de trabajo por sexo, incorporándose el tiempo de trabajo en cada caso, lo cual se relaciona con el rol cultural adosado a la mujer como cuidadora del hogar y la familia. Esta visión de sociedad es, además, profundizada cuando ambos padres tienen una carrera profesional a la cual se deben dedicar (McPherson, 2010).

Las mujeres que participan en los consejos superiores, deben continuar con sus labores académicas de docencia, investigación, vinculación con el medio y gestión directiva. Sin embargo, la carga de trabajo se ha visto aumentada debido a la pandemia y el teletrabajo, muchos hogares debieron prescindir de la ayuda doméstica, lo cual impactó sobre los tiempos destinados a las labores del hogar y enfatizó la baja corresponsabilidad que existe en las familias, donde la mujer es quien está a cargo del cuidado de la familia y las cosas del hogar. (comunicación personal E1, 2020) “A pesar de que estábamos todos en casa, se da por hecho que la mamá debe realizar las cosas para que funcione la casa, así realmente no me pude concentrar en un artículo que tengo pendiente". (comunicación personal E3, 2020) "Los alumnos no entienden que todos necesitamos descansar, no les importa la hora, ellos quieren atención en cualquier momento". (comunicación personal E9, 2020) "Las cuarentenas realmente han sido nefastas para mí, además de todo el trabajo de la universidad, debo realizar las tareas domésticas". 


\section{Journal of the Academy | 41 |}

Las barreras principalmente se destacan desde un punto de vista cultural, argumentando que no todas las mujeres están dispuestas a trabajar de manera gratuita, además de una cultura aún patriarcal, donde se asignan ciertos roles familiares a las mujeres, a saber, el cuidado y la crianza de los hijos y trabajos domésticos.

(comunicación personal E7, 2020) “culturalmente creo que no todas las mujeres están dispuestas a dedicar días completos gratuitamente a una labor de este tipo, si están dispuestas muchas mujeres a trabajar gratuitamente en muchas cosas pero que tienen que ver con el ámbito reproductivo familiar no con el ámbito profesional o académico...",

(comunicación personal E2, 2020) "Realmente no comprendo por qué envían tantos documentos para revisar con tan poco tiempo, así es difícil poder descansar el tiempo necesario". Es decir, con respecto al rol familiar por género, todavía se atribuye la responsabilidad de la crianza de los niños principalmente a las madres y no a los padres (Arana y Carrillo, 2016). De este modo, se puede inferir que las mujeres dedican más tiempo al ámbito familiar que al ámbito laboral, lo cual en puestos de poder las deja en clara desventaja.

Los tres niveles que se analizaron anteriormente en relación a la participación de las mujeres en cargos de alta dirección universitaria, individual, organizacional y cultural, inciden en las decisiones que toman las mujeres respecto de su participación en los máximos cuerpos colegiados. A nivel individual, las mujeres que deciden participar en los Consejos Superiores, sienten una gran responsabilidad y orgullo de pertenecer a este órgano, pero también existen temores y falta de seguridad que frenan su desarrollo. A nivel organizacional, un elemento a considerar es la progresión académica, lo cual muchas veces juega en contra de sus aspiraciones. Finalmente, a nivel cultural, aún existe un gran peso social respecto a estereotipos asociados a la mujer.

\section{CONCLUSIONES}

El objetivo principal de este estudio ha sido analizar los factores que inciden en la baja participación de las mujeres en las juntas directivas de las universidades chilenas. A lo largo de la investigación se ha realizado un análisis de la situación de la mujer respecto a los altos cargos, ya que en la mayoría de las universidades en el nivel más alto de su estructura existe menos presencia de mujeres que en niveles de menor jerarquía. Así mismo a través de la información obtenida de las páginas web de cada institución se establece que actualmente existe un $31 \%$ de los cargos ocupados por mujeres en las juntas directivas o Consejos superiores de las universidades estatales o católicas, respectivamente. Lo cual principalmente se debe a las barreras que las mujeres 


\section{Journal of the Academy | 42 |}

enfrentan, no sólo dentro de una organización para acceder a cargos de mayor responsabilidad, sino también a nivel personal (individual) y a nivel cultural y social.

A nivel individual, se logró corroborar que estas barreras invisibles se relacionan principalmente por las bajas expectativas que tiene la mujer sobre sus capacidades, por miedo al riesgo, baja seguridad en sí mismas y bajo deseo de poder, deduciendo de cierta forma la existencia de techos de cristal (Del Pino Arriagada et al., 2018). Esto debido a los estereotipos que configuran el que las mujeres no estén en la junta directiva destacando que "las mujeres temen tener posiciones de poder"; "No les interesa tener mayor responsabilidad". Debido a las características de los cargos de la junta directiva, la trayectoria profesional es un factor importante en las personas que la conforman, es decir tanto para hombres como para mujeres ya que son relevantes los conocimientos y la experiencia que pueda tener cada persona, esto debido a la cantidad de decisiones que se deben tomar en una reunión y la responsabilidad que se requiere para ejercer estos cargos, lo cual coincide con otras investigaciones que dicen que las decisiones y las acciones que las universidades implementan para cumplir sus objetivos se basan principalmente en las capacidades y habilidades de las personas que ocupan puestos de alta dirección. En las juntas directivas y consejos superiores, las decisiones se toman en conjunto por medio de votaciones, y el punto de vista tanto de hombres como mujeres, es valorado y necesario, sobre todo en las universidades, donde las decisiones que se toman, afectan no sólo a quiénes trabajan allí sino al alumnado y a la sociedad. Esto demuestra que la falta de mujeres líderes no es por un tema de capacidades y conocimientos, sino que es por un tema principalmente cultural, que les impide a las mujeres estar en cargos superiores. Por ello es importante que las mujeres superen estas barreras invisibles y accedan a los altos cargos para así tener una exitosa carrera profesional.

Hay un tema sobre el cual se debe llamar la atención, y es en relación a la categorización o jerarquización académica, la cual está siendo una barrera indirecta para que las mujeres puedan optar a cargos directivos, ya sea porque es necesario pertenecer a las categorías o jerarquías más altas de la academia o porque al optar por un cargo de gestión o alta dirección, se debe postergar el ascenso de categoría, el cual ya sufre postergaciones por aspectos personales, como es la maternidad y crianza de los hijos.

Por consiguiente, según lo analizado anteriormente se tiene como resultado que los factores que influyen en la participación femenina en los cargos de la junta directiva de las universidades 


\section{Journal of the Academy | 43 |}

chilenas se debe principalmente a las barreras invisibles que enfrentan las mujeres, relacionadas con la teoría del techo de cristal (miedo al riesgo, a los desafíos) y por el rol que cumplen dentro de la familia asociándolo con la teoría del suelo pegajoso, en donde lo primordial es la vida familiar y no el desarrollo profesional.

Futuras investigaciones, debiesen orientarse a analizar factores que aporten al empoderamiento de las académicas en roles de toma de decisiones, y además la búsqueda y desafío para mejorar la progresión académica, de manera de aumentar la cantidad de mujeres en las más altas categorías,

y de esta manera mejorar su posición para acceder a los máximos cuerpos colegiados, como es el Consejo Superior, y desde allí aportar con una visión diferente a las alternativas de decisiones que se deben tomar.

\section{REFERENCIAS}

Adu-Oppong, A.; Aikins, E. y Darko, G. (2017). The place of women in higher education management: a Ghanaian perspective. Global Journal of Interdisciplinary Social Science, (6)1, $10-18$

Aslanargum, E. (2013). Stereotypes and barriers for woman principals in differents cultures, NWSA Education Science 8(4), 460-470.

Aequalis (2017). "Participación femenina en cargos directivos en instituciones de educación superior chilena". Aequalis, Foro de educación superior. Santiago, Chile, disponible en http://www.aequalis.cl (consultado 15 de April, 2018).

Arana, K., y Carrillo, S. (2016). Diagnóstico cualitativo sobre los factores que condicionan la participación de las mujeres en puestos laborales de liderazgo y su vinculación al plan nacional de igualdad de género. [Tesis de Magister., Pontificia Universidad Católica del Perú]. Repositorio Institucional. https://tesis.pucp.edu.pe/repositorio/handle/20.500.12404/7141

Barberá, E., Ramos, A., Sarrió, M., y Candela, C. (2011). Más allá del “techo de cristal” Diversidad de género. Revista del Ministerio de trabajo y asuntos sociales, (40), 55- 68.

Brandt, M. y Edinger, P. (2015). Transformational leadership in teams-the effects of a team leader's sex and personality. Gender in Management: An International Journal, 30(1), 4468. https://doi.org/10.1108/GM-08-2013-0100

Camarena, M., y Saavedra, M. (2018). El techo de Cristal en México. Revista de estudios de género, 47, 312- 347. 


\section{Journal of the Academy | 44 |}

Creswell, J. (2007. Qualitative inquiry and research design: Choosing among five approaches. (2nd ed.).Sage Publications Inc.

Del Pino, S., Vallejos, R., Améstica- Rivas, L. y Cornejo- Saavedra, E. (2018). Presencia de las mujeres en la alta gestión universitaria. Las universidades públicas en Chile. Revista páginas de educación, 11(2), 176- 198.

Del Pino, S. (2015). El rol de la mujer en el desempeño y gestión universitaria de las instituciones estatales de educación superior de Chile.[Tesis de Magíster, Universidad del Biobío]. Repositorio

Institucional. http://repobib.ubiobio.cl/jspui/bitstream/123456789/1693/1/Pino\%20Arriagada\%20Sara\%20d el.pdf

Dezsö, C., y Gaddis, D. (2007). Girl power: Female participation in top management and firm performance. Tesis de magister, Facultad de negocios, Universidad de Maryland. Maryland, EE.UU. 34 pp.

Díaz, Y., Andrade, J., y Ramírez, E. (2019). Liderazgo Transformacional y Responsabilidad Social en Asociaciones de Mujeres Cafeteras en el Sur de Colombia. Información tecnológica, 30(5), 121-130. https://dx.doi.org/10.4067/S0718-07642019000500121

Dinh, J., Lord, R., Gardner, W., Meuser, J., Liden, R. y Hu, J. (2014). Leadership theory and research in the new millennium: Current theoretical trends and changing perspectives. The leadership quarterly 25(1), 36-62.

Duarte, L. (2012). La presencia de las mujeres en los órganos de toma de decisiones y dirección en la UAB. Observatori per a la igualtat de la UAB, 41- 71. https://core.ac.uk/download/pdf/13324911.pdf

Durán-Seguel, I., Gallegos, M., y Cabezas, D. (2019). Estilos de liderazgo y su influencia en el clima laboral: caso de estudio de una empresa exportadora de alimentos. Revista ESPACIOS, 40(40), 3-14.

Foro Económico Mundial (2020). Organización internacional para la cooperación público-privada. https://es.weforum.org/

Gaete, R. (2015). El techo de Cristal en las Universidades estatales chilenas. Un análisis exploratorio. Revista Iberoamericana de Educación superior, 6(17), 3- 20. 


\section{Journal of the Academy | 45 |}

Gaete, R. y Álvarez, J. (2020). Alta dirección pública y techo de cristal. Acceso de las mujeres a los puestos directivos en Chile. Espiral (Guadalajara), 27(77), 179-222. Epub 12 de febrero de 2021. https://doi.org/10.32870/eees.v27i77.7085

Gallego, N. (2017). Breaking the glass ceiling. Metode science studies journal, 7, 113- 119.

Gallegos, F., Guzmán, J., Saavedra, C., y Silva, A. (2012). Participación de mujeres en cargos gerenciales: Una investigación cualitativa de empresas financieras. [Tesis de grado, Universidad de Chile]. Repositorio Institucional.

https://repositorio.uchile.cl/bitstream/handle/2250/111789/Participaci\%c3\%b3n\%20de\%20M ujeres\%20en\%20Cargos\%20Gerenciales.pdf?sequence=1\&isAllowed=y

García, R., y Lopez, E. (2006). Congruencia de rol de género y liderazgo: El papel de las atribuciones causales sobre el éxito y el fracaso. Revista Latinoamericana de Psicología, 38(2), 245- 257.

Gutiérrez, O. (2015). Estudios de liderazgo de hombres y mujeres. Revista política y estrategia, $126,133-171$.

Hernandez, A., Escartín, J., y Van Dick, R. (2014). Gender and leadership in Spain: A systematic review of some key aspects. Sex Roles, 70(11-12), 522-537. https://doi.org/10.1007/s11199$\underline{014-0375-7}$

Lupano, M. y Castro, A. (2013) Estudios sobre el liderazgo. Teorías y evaluación. Psicología, Cultura y Sociedad, (6), pp.107-121.

McPherson, M. (2010). Women in senior management why not? Labour, employment and work in New Zeland, 1-11. https://doi.org/10.26686/lew.v0i0.1718

Maak, T., Pless, N. y Voegtlin, C. (2016) Business Statesman or Shareholder Advocate? CEO Responsible Leadership Styles and the Micro-Foundations of Political CSR, Journal of Management Studies, 3(53), 463-493, https://doi.org/10.1111/joms.12195

Maheshwari, G. y Nayak, R. (2020) Women leadership in Vietnamese higher education institutions: An exploratory study on barriers and enablers for career enhancement, Educational Management Administration \& Leadership, 1-18. https://doi.org/10.1177/1741143220945700

Matteo, C. A. (2012). La mujer en el entorno gerencial venezolano: perfil, características y desempeño. Revista Venezolana de Estudios de La Mujer, 17(38), 39-66.

Matus, M., y Gallego, N. (2015). Techo de Cristal en la Universidad. Si no lo veo no lo creo. Revista Complutense de Educación, 26(3), 611- 626. 


\section{Journal of the Academy | 46 |}

Méndez, R., y Rojas, M. (2014). El género: una variable invisible pero sustantiva en la educación superior. Sophia, 10(1), 74-82.

Mendoza, I., Escobar, G., y García, B. (2012). Influencia del liderazgo transformacional en algunas variables de satisfacción organizacional en personal docente y administrativo de una institución pública de educación media superior. Revista Del Centro De Investigación De La Universidad La Salle, 10(38), 189-206. https://doi.org/10.26457/recein.v10i38.92

Moncayo, B. y Pinzón, N. (2013). Women leaders in academia, gender and stereotypes. Panorama, 7(13), 75-94

Papadópulos, J., y Radakovich, R. (2006). Educación Superior y género en América Latina. En Instituto Internacional de la UNESCO para la Educación Superior en América Latina y el Caribe (IESALC). Informe sobre la educación superior en América Latina y el Caribe 20002005. La Metamorfosis de la Educación Superior y género en América Latina. (117-128). Editorial Metrópolis. $\quad$ http://www.sela.org/media/3202539/t023600002757-0informe_sobre_la_educaci\%C3\%B3n_superior_en_al_2000-2005.pdf

Peiying Chen y Hsiao-chin Hsieh (2019) Women's academic leadership under competing higher education policies in Taiwan, Compare. A Journal of Comparative and International Education, 49(5), 759-776, https://doi.org/10.1080/03057925.2018.1454826

Perozo, B., y Paz, A. (2016). Estilos de liderazgo femenino como factor influyente en las habilidades de los equipos de trabajo en el sector asegurador. Clío América, 10(19), 8-22. https://doi.org/10.21676/23897848.1656

Ricks, H. (2018). Ascending: An Exploration of Women's Leadership. Advancement in the Role of Board of Trustee Chair. Administrative Science, 1-12

Serrano, B. y Portalanza, A. (2014) Influencia del liderazgo sobre el clima organizacional. Suma de Negocios, 5(11), 117-125.

Shepherd, S. (2017). Why are there so few female leaders in higher education: A case of structure or agency? Management in Education, $31(2), \quad 82-87$; https://doi.org/10.1177/0892020617696631

Tongco, D. (2007). Purposive Sampling as a Tool for Informant Selection. Research and Applications, 5, 147-158. https://ethnobotanyjournal.org/index.php/era/article/view/126

Torres, O., y Pau, B. (2011). Techo de cristal y suelo pegajoso. La situación de la mujer en los sistemas alemán y español de ciencia y tecnología. Revista CTS, 6(18), 35-39. 


\section{Journal of the Academy $|47|$}

Vázquez, S., Bernal, J. y Liesa, M. (2014). La conceptualización del liderazgo: una aproximación desde la práctica educativa. Revista iberoamericana sobre Calidad, Eficacia y Cambio en Educación, REICE12(5), 79-97.

Vaca, I. (2019) Oportunidades y desafíos para la autonomía de las mujeres en el futuro escenario del trabajo.(Serie Asuntos de Género, $\mathrm{N}^{\circ}$ 154). Comisión Económica para América Latina y el Caribe (CEPAL) en Santiago de Chile. https://repositorio.cepal.org/bitstream/handle/11362/44408/4/S1801209_es.pdf

Vinkenburg, C., Van Engen, M ., Eagly, A y Johannesen, M (2011). An exploration of stereotypical beliefs about leadership styles: Is transformational leadership a route to womwn's promotion? The Leadership Quarterly, 22(1), 10-21. https://doi.org/10.1016/j.leaqua.2010.12.003

Wylie, A., Jakobsen, J. R., y Fosado, G. (2007). Women, work, and the academy. The Barnard Center for Research on Women, 2,1-17 